\title{
Fourier transform infrared spectroscopy of a single aerosol particle
}

\author{
Gideon Sageev Grader ${ }^{\text {a) }}$ \\ Department of Chemical Engineering, California Institute of Technology, Pasadena, California 91125 \\ Stephen Arnold \\ Department of Physics, Polytechnic Institute of New York, Brooklyn, New York 11201 \\ Richard C. Flagan and John H. Seinfeld \\ Department of Chemical Engineering, California Institute of Technology, Pasadena, California 91125
}

(Received 28 November 1986; accepted 17 February 1987)

\begin{abstract}
A method is developed for obtaining the molecular composition of a single suspended microparticle by Fourier transform infrared spectroscopy. The particle is held in an electrodynamic balance and irradiated simultaneously by the infrared output from a Michelson interferometer and the visible light from a dye laser. The laser is tuned to an edge of an optical resonance of the particle while the IR beam is chopped. Through evaporation and condensation the chopped IR beam causes a size modulation of the droplet, which in turn induces a fluctuation in the laser light scattered from the particle. The scattered light is detected at $90^{\circ}$ with a photomultiplier, and the amplitude of the light fluctuation is measured with a lock-in amplifier. The lock-in signal is then inverted by a discrete fast Fourier transform routine (FFT), to yield the particle absorption spectrum. Spectra of $\left(\mathrm{NH}_{4}\right)_{2} \mathrm{SO}_{4}$ droplets at different solute concentrations are presented. The data shown include the first infrared spectrum of a highly supersaturated solution.
\end{abstract}

\section{INTRODUCTION}

A long-standing goal in aerosol science has been to develop methods to probe the molecular composition of a single microparticle. One approach to isolate a single particle is to use an electrodynamic balance ${ }^{1-3}$ (also called a quadrupole), within which a micron-sized, charged particle can be levitated virtually indefinitely. The electrodynamic balance offers the additional advantage of affording a well-controlled gaseous environment for studying the response of the particle to temperature variations and changes in its surrounding vapor. For a liquid particle, e.g., the lack of physical contact with foreign surfaces and other particles makes the system very suitable for the study of solution-phase phenomena such as vapor-liquid equilibrium, ${ }^{4,5}$ particle-phase chemical reactions, ${ }^{6}$ and deliquescence crystallization. ${ }^{7}$

A number of methods have recently been reported for determining the molecular composition of a single liquid particle including ones based on the fluorescence ${ }^{8}$ and $\mathrm{Ra}-$ $\operatorname{man}^{9,10}$ emission from the particle and on the infrared absorption $^{11,12}$ of the particle. When applied to a micron-sized particle, the excitation wavelength in both the fluorescence and Raman techniques is small compared with the particle diameter. Therefore the spectra, obtained via the scattered light from the particle, are complicated by the resonant nature of the scattering in the Mie regime. The spectra may also be affected by a nonhomogeneous distribution of scatterers inside the particle. In contrast, when infrared light is used to excite the particle, the wavelength is comparable with the particle size. Therefore under this condition the particle will appear almost Rayleigh like, ${ }^{12}$ where the absorption is uniform and can be related to the average composition.

The absorption spectrum of an aqueous droplet may be

\footnotetext{
a) Current address: AT\&T Bell Laboratories, Murray Hill, NJ 07974.
}

obtained by heating with an IR source, and simultaneously illuminating the droplet with a visible laser. ${ }^{11}$ When an aqueous solution droplet is suspended in a humid environment its water activity equals the ambient relative humidity and, as long as the relative humidity is held constant, the particle size remains constant (provided the solute in the drop is nonvolatile). Upon heating, the droplet evaporates due to the increase of the water vapor pressure with temperature. This evaporation is restrained, however, by the increase in the ionic strength in the particle accompanying water loss. When the heating is stopped, the particle returns to its initial temperature and its original size. Therefore, periodically exposing the particle to an infrared beam will cause a periodic fluctuation in the particle size.

Since the visible scattered light from a particle is a strong function of its size, size fluctuations cause a corresponding modulation in the scattered light from the particle. Arnold et al..$^{1-13}$ have shown that this modulation may be enhanced if the light scattering is measured near a structure resonance of the particle. If, in addition, the particle is heated by selected wavelengths in the IR, larger modulations in the size and light scattering will occur at wavelengths where species in the particle absorb the incident radiation. By correlating the magnitude of the scattered light fluctuation with the incident IR wavelength, the absorption spectrum of the particle can be obtained. In Arnold, Neuman, and Pluchino's technique ${ }^{11}$ the wavelength in the IR was selected with a wedge monochromator. The advantages of this technique are its simplicity, and ability to monitor the absorption at a particular wavelength continuously. However the spectral resolution is limited to the bandwidth at a given position of the wedge. In addition, the monochromator acts as a filter which limits the amount of intensity delivered to the particle. 
This paper introduces a Fourier transform technique for obtaining the composition of a single suspended aerosol particle similar to that proposed by Arnold and Pluchino. ${ }^{12}$ In the present technique the entire IR beam is passed through a Michelson interferometer consisting of a beam splitter and two mirrors. At the beam splitter different wavelengths undergo constructive and destructive interference depending on the relative separation between the mirrors and the beam splitter. As a result the FTIR technique offers a greater energy throughput to the sample. Since the resolution in FTIR spectroscopy is inversely proportional to the scan length of the interferometer mirror, this method can reach higher resolution than the previous technique. For example, a $1 \mathrm{~cm}$ mirror scan can potentially yield a resolution of $1 \mathrm{~cm}^{-1}$, which is an order of magnitude smaller than the resolution of the wedge monochromator. Although the added resolution has a limited application in aqueous solutions in which the vibrational resonances are broad ${ }^{14}\left(\geqslant 50 \mathrm{~cm}^{-1}\right)$, in future applications the higher potential resolution could prove useful.

In the next section the theory of the FTIR technique will be developed. Then the apparatus and experiment will be described. Finally results of application of the technique to ammonium sulfate droplets will be presented.

\section{THEORY}

The IR spectroscopy developed here is based on two fundamental processes: evaporation and condensation due to particle heating and cooling, and fluctuations in the light scattering resulting from particle size changes. The evaporation and subsequent relaxation back to its original size of a particle momentarily heated can be used to provide data on the simultaneous heat and mass transfer processes in the continuum ${ }^{13}$ as well as the transition ${ }^{15}$ regime. In such processes, if the size change is small relative to its initial size, for a particle with an initial radius $a_{0}$, the perturbation in the radius $\epsilon(t)=a(t)-a_{0}$ can be shown to be governed by ${ }^{13,15}$

$$
\frac{d \epsilon}{d t}=-\int_{0}^{\infty} \alpha(k) I_{0}(k) d k-\gamma \epsilon,
$$

where $I_{0}(k) d k$ is the incremental IR intensity at wave number $k . \alpha(k)$ is related to the particle absorption coefficient $Q_{a}(k)$ and is given by

$$
\alpha(k)=\frac{Z}{L Z+1} \frac{Q_{a}(k)}{4 \rho_{p}},
$$

where $L$ and $\rho_{p}$ are the water latent heat of vaporization and the particle density, respectively, and $Z$ is a constant that depends on the water diffusivity in the gas, the heat transfer properties of the particle, and the salt concentration. Finally, $\gamma$ in Eq. (1) is the particle relaxation rate (when the heating is stopped), and is given by

$$
\gamma=\frac{3 D_{g} C^{0} i X_{w}\left(1-X_{w}\right)}{\rho_{p} a_{0}^{2} f_{w}(1+L Z)},
$$

where $D_{g}$ and $C^{0}$ are the water diffusivity in the gas, and the pure water vapor pressure, respectively. $X_{w}$ and $f_{w}$ are the water mole fraction and weight fraction in the droplet, respectively, and $i$ is the Van't Hoff factor.
The incremental radius change resulting from heating and cooling, $\epsilon(t)$, can be related to a fluctuation, $\delta S(t)$, in the scattered light from the particle in terms of an amplification factor $\beta,{ }^{12}$

$$
\frac{\delta S(t)}{S_{0}}=\beta \frac{\epsilon(t)}{a_{0}},
$$

where $S_{0}$ is the scattering signal from the particle before the size modulation.

If the infrared source is from a Michelson interferometer, the incremental intensity in the wave number range $(k, k+d k)$ at a mirror distance $l$ from its equal arm point is given by

$$
I_{0}(l, k) d k=e^{j 2 k l} T(k) I_{s}(k) d k,
$$

where the wave number of the infrared light is related to its wavelength by, $k=(2 \pi / \lambda), T(k)$ is the transmission function of the optical elements in the IR pathway, between the source and the particle, and $I_{s}(k) d k$ is the incremental source intensity between $k$ and $k+d k$. If the output intensity is chopped at a frequency $\omega$, Eq. (5) becomes

$$
I_{0}(l, k, t) d k=e^{j(2 k l+\omega t)} T(k) I_{s}(k) d k .
$$

The time dependent IR excitation can be employed to drive the particle size change as expressed in Eq. (1). Note that the total intensity received by the particle is the integral of Eq. (6) over all wavelengths. Since the particle responds at the same frequency as the driving heat source, the solution to Eq. (1) can be expressed as

$$
\epsilon(t, l)=\int_{-\infty}^{\infty} A(k) e^{j(\omega t+\phi)} d k
$$

where $\phi$ is the phase of the particle response with respect to the driving force. Substituting Eqs. (5) and (7) into Eq. (1) yields the values of $A(k)$ and $\phi$. The resulting size fluctuation is given by

$$
\epsilon(t, l)=-\int_{-\infty}^{\infty} \frac{\alpha(k) I_{s}(k) T(k)}{\left(\omega^{2}+\gamma^{2}\right)^{1 / 2}} e^{j(\omega t+\phi)} d k,
$$

where $\phi(k)=2 k l-(\omega / \gamma)$. The change in the radius is seen to be a function of the mirror position and time. Using Eq. (4), the corresponding scattered light fluctuation is

$$
\frac{\delta S(t, l)}{S_{0}}=-\int_{-\infty}^{\infty} \frac{\beta}{a_{0}} \frac{\alpha(k) I_{s}(k) T(k)}{\left(\omega^{2}+\gamma^{2}\right)^{1 / 2}} e^{j(\omega t+\phi)} d k .
$$

The equation above represents the incremental time dependent scattering signal resulting from the chopped IR heating of the particle. We desire the magnitude of the ac component of the scattered light fluctuation. To obtain a dc level from Eq. (9) we detect the scattered light signal with a lock-in amplifier, referenced to the IR chopper frequency. At the output of the lock-in amplifier the scattered light signal at any mirror position $l$ is

$$
\frac{\langle\delta S(t, l)\rangle}{S_{0}}=-\int_{-\infty}^{\infty} B(k) e^{j(k l+\theta)} d k,
$$

where

$$
B(k)=\frac{\beta}{a_{0}} \frac{\alpha(k) I_{s}(k) T(k)}{\left(\omega^{2}+\gamma^{2}\right)^{1 / 2}},
$$

$\theta=-(\omega / \gamma)$, and $\langle\delta S(l)\rangle$ is the measured signal in the 
present experiment. The chemical information that is of interest is in the absorption coefficient $\alpha(k)$, which is contained in $B(k)$. Multiplying Eq. (10) by $e^{-j k^{\prime} l} d l$ and integrating over all mirror positions yields

$$
\frac{1}{2 \pi} \int_{-\infty}^{\infty} \frac{\langle\delta S(l)\rangle}{S_{0}} e^{-j k^{\prime} l} d l=B\left(k^{\prime}\right)
$$

which is recognized as the inverse Fourier transform of the data which can be carried out with a discrete fast Fourier transform (FFT) routine. ${ }^{16}$ Since $\gamma$ and $\omega$ are known constants, and $I_{s}(k)$ and $T(k)$ are determined for the particular source and interferometer optics, the evaluation of $B(k)$ allows us to obtain $\alpha(k)$ which depends directly on the particle absorption coefficient $Q_{a}(k)$.

\section{EXPERIMENTAL SYSTEM}

In this experiment a single charged particle is held by the electric field generated with a quadrupole trap. As shown in Fig. 1, the trap consists of top and bottom electrodes between which a dc voltage is held, and a ring electrode to which an ac voltage is applied. The exact geometry of the trap has been described earlier, ${ }^{15,17}$ and for now it suffices to know that the device can hold a charged particle virtually indefinitely. The historical development of this technique and the behavior of charged particles in its electric field have been documented elsewhere. ${ }^{1-3}$ The trap is encased inside a sealed chamber that can be evacuated and filled back with the gases of interest. Particles are introduced into the chamber by opening the top seal and lowering a particle jet above the top electrode. The jet (Uni Photon System model 1) works on the following principle. ${ }^{18} \mathrm{By}$ applying an electrical pulse to a piezoelectric crystal, a pressure wave is generated that forces a droplet out a small opening in the glass tip (see Fig. 1). The droplet is charged inductively as it leaves the tip of the jet and is then trapped by the ac field. The whole jet assembly is enclosed inside a Teflon bag through which filtered dry air is continuously passed. In so doing we prevent room-air particles from entering the chamber and contaminating the particle. The relative humidity inside the chamber is controlled through a connection of the chamber to a water reservoir positioned in a constant temperature bath.

A schematic of the experimental system is shown in Fig. 2, where the electrodes and chamber are omitted for the sake of clarity (the particle in the figure is not drawn to scale). In describing the apparatus the IR pathway will first be traced, and then the laser and associated electronics will be discussed. The infrared beam is generated in a commercial Michelson interferometer (Advanced Kinetics), and consists of a $2 \mathrm{~mm}$ wide glowbar (Perkin Elmer), collimated by $f / 3$ optics. The instrument is supplied with a geared-down dc motor coupled to a micrometer. The motion of the mirror is followed with a Moire encoder. The interferometer is designed to scan continuously, however, the instrument was modified to step the mirror in equal intervals, allowing integrating of the signal at each of the mirror positions. The reason for this modification is that at each mirror position time must be allowed for the droplet to relax to its preheated size. If the interferometer mirror is driven continuously

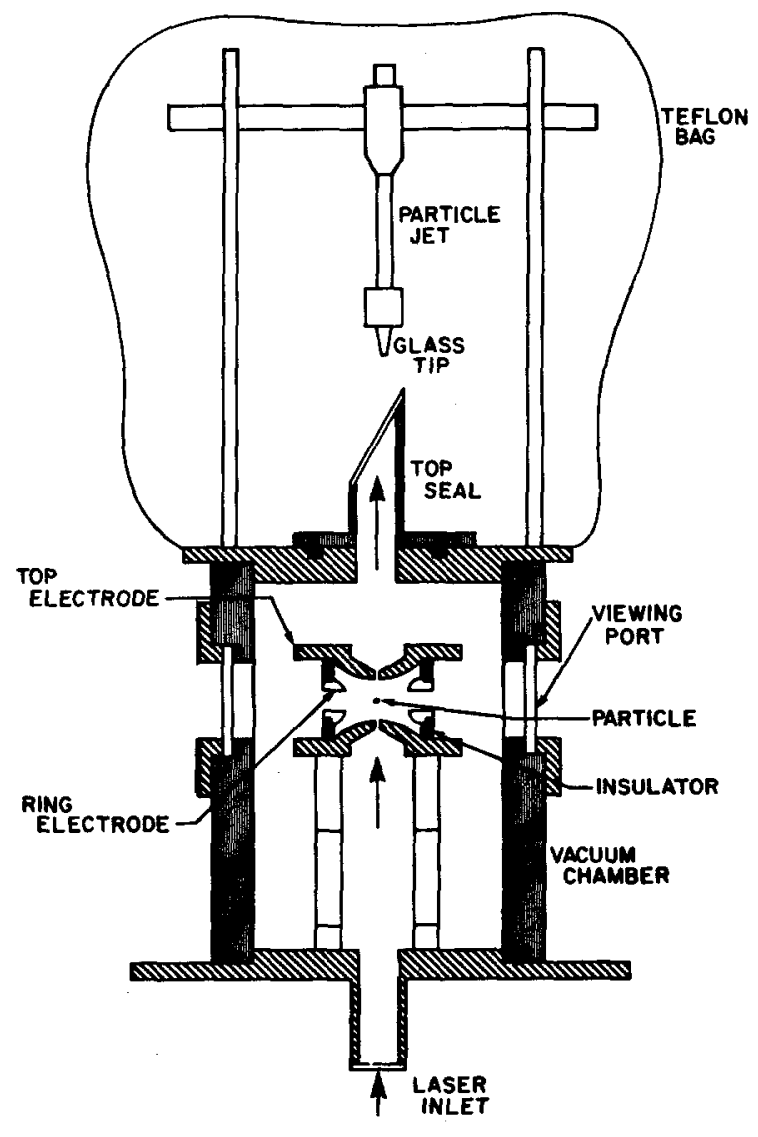

FIG. 1. A schematic diagram of the single particle levitation system, the vacuum chamber, and the particle jet.

(without the chopper), the rate of the IR signal modulation depends on the mirror speed. For example at the slowest mirror speed of $10 \mu \mathrm{m} \mathrm{s}^{-1}$ and wavelength of $5 \mu \mathrm{m}$, an IR fringe will pass every $0.5 \mathrm{~s}$. Recording the scattered light level at every micron of the mirror travel will allow $100 \mathrm{~ms}$ between readings. Under this condition not only must the droplet respond at a faster rate, but also the signal-to-noise ratio must be sufficiently large in that bandwidth. For the particles considered in this study a 10-30 s integration time constant was needed to obtain a sufficiently clean signal. Being able to step the interferometer mirror and chop the IR was critical as it enabled signal averaging for as long as necessary.

The output beam is first focused on an iris and then chopped at $5-10 \mathrm{~Hz}$. Since the particle absorbs only a minute fraction of the beam, preventing the major part of the beam from entering the system avoids excessive heating of the chamber. This fact is important in maintaining the relative humidity surrounding the particle constant during the experiment. The diverging beam is then refocused on the particle. After passing the particle, the beam exits through an opposite window. The mercury cadmium teluride (MCT) detector shown in line with the IR beam is used for the initial alignment and continuous monitoring of the interferometer output during an experiment.

Simultaneously with the IR beam the particle is also illuminated with a visible dye laser in the vertical direction. 


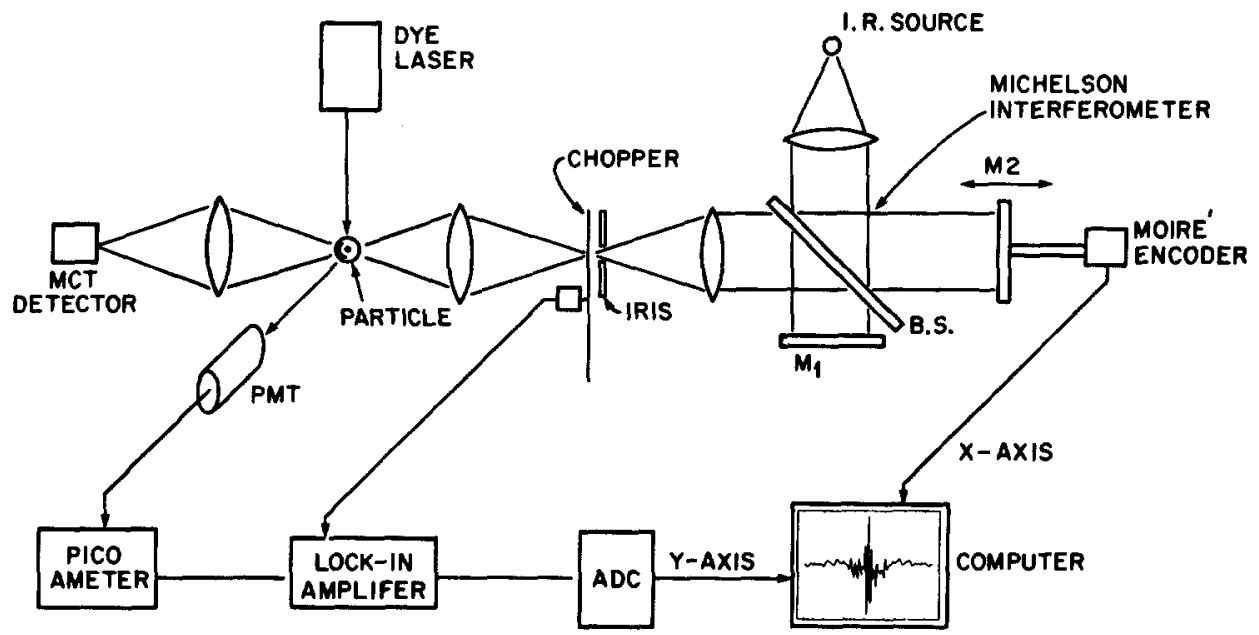

FIG. 2. The experimental layout for single particle Fourier transform infrared spectroscopy.
The dye laser used is a Spectra Physics model 375, pumped with their argon ion laser model $2020-5$. The $90^{\circ}$ visible scattered light from the particle is collected through a microscope objective and coupled into a photomultiplier tube. The PMT signal is measured with a picoammeter and is then fed to the input stage of a lock-in amplifier. As mentioned earlier, the PMT signal contains a fluctuation resulting from the size modulation of the droplet. The amplitude of this fluctuation is measured by referencing the lock-in to the chopper output. The lock-in output is sent to a 16-bit A/D converter and read by a computer. As shown, the computer (Zenith 120) also synchronizes the stepping of the interferometer mirror. Depending on the lock-in time constant, the computer is programmed to allow enough time between moving the mirror and taking a new $A / D$ reading. At the end of a scan the data are inverted by FFT to yield the absorption spectrum.

In this experiment we make use of the optical resonances occurring in the scattered light from a small particle. An example of such resonances for a $\left(\mathrm{NH}_{4}\right)_{2} \mathrm{SO}_{4}$ particle is shown in Fig. 3. These resonances were predicted by $\mathrm{Mie}^{19}$ and were first observed by Ashkin and Dziedzic. ${ }^{20}$ For a given particle of radius $a$, and incident radiation of wavelength $\lambda$, the optical parameter $(2 \pi a / \lambda)$ remains constant for a particular resonance. It then follows that pertubations in the radius $\delta a(=\epsilon)$ and in the wavelength $\delta \lambda$ for such a resonance are related by $(\delta a / a)=\cdot(\delta \lambda / \lambda) .{ }^{11}$ Therefore, in a region where the scattered light changes linearly with wavelength (i.e., on the edge of an optical resonance), it also changes linearly with the size fluctuations of the particle.

It is critical that during an experiment, we remain on the linear portion of the scattering curve so that size fluctuations are linearly related to the scattered light fluctuation. At the start of an experiment we first tune the dye laser to the edge of an optical resonance (see Fig. 3), thereby turning the resonance into an optical amplifier for small size changes in the droplet. The amplification factor $\beta$ in Eq. (2) can exceed 4000. ${ }^{11}$ Once the laser is tuned, the chopper is turned on and the scattered light fluctuations are observed on an $X-Y$ recorder. Small drifts in the particle size due to changes in the relative humidity in the chamber as a result of slow heating of the chamber will cause the system to drift off the edge of the resonance. These drifts are compensated by retuning the dye laser. In this fashion the amplification factor $\beta$ remains constant during an experiment.

While scanning the interferometer the mirror is moved in equal steps from one side of the equal arm point to the other. The lock-in time constant normally used was 10 s; and 140 data points were usually taken each scan, separated by roughly $1 \mu \mathrm{m}$ and centered about the equal arm point. Increasing the resolution by taking longer scans did not prove useful because of low signal-to-noise ratio in the wings of the interferogram. This problem could be avoided by increasing the lock-in time constant. We have used a $30 \mathrm{~s}$ time constant, however, increasing it any further would make the time necessary for a single scan prohibitively large. It should be pointed out that after turning the system on, we allow about $1 \mathrm{~h}$ for all the components to stabilize before taking the first scan.

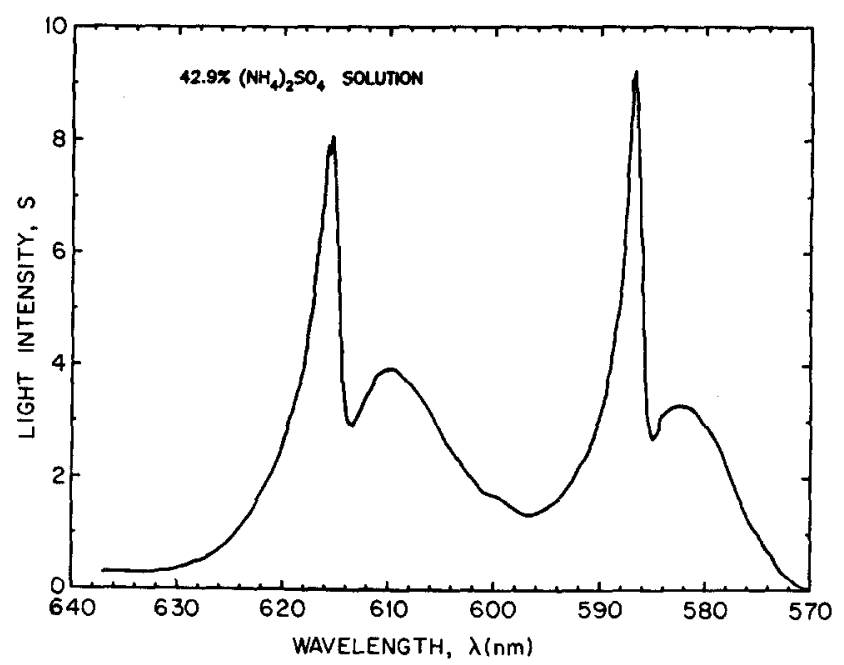

FIG. 3. The scattered light intensity at $90^{\circ}$ from a single $\left(\mathrm{NH}_{4}\right)_{2} \mathrm{SO}_{4}$ particle, illuminated by a dye laser, as a function of the laser wavelength. 


\section{RESULTS AND DISCUSSION}

An example of the recorded scattered light signal $\langle\delta S(l)\rangle$, as a function of mirror position, is shown in Fig. 4. The zero point on the $x$ axis corresponds to the position where the two interferometer mirrors are at equal distances from the beam splitter. Only at this position are all the waves recombining at the beam splitter in phase with each other. Therefore, at this point all those bonds of species inside the droplet absorbing in the IR are excited. This gives rise to a maximum size modulation signal at the equal arm point. Hence at this position the scattered light modulation reaches the maximum shown in Fig. 4. As the mirror is moved on either side of the equal arm point, some wavelengths undergo destructive interference at the beam splitter, giving rise to a smaller absorption that is reflected in a smaller scattered light fluctuation. As the mirror is moved further from the equal arm point some of the waves combine at the beam splitter incoherently. The incoherent recombination reduces the intensity modulation at all wavelengths which shows up as smaller modulation signals in Fig. 4. For the scan shown in Fig. 4, the particle consisted of a $24.4 \%$ (by mass) aqueous solution of ammonium sulfate. The particle radius was $3.14 \pm 0.03 \mu \mathrm{m}$. The data shown in Fig. 4 represent the first infrared interferogram reported for a single aqueous droplet.

By inverting the data in Fig. 4 by FFT, the resulting $B(k)$ spectrum shown in Fig. 5 is obtained. From Eq. (11) we note that $B(k)$ contains the instrument line function $T(k) I_{s}(k) . T(k)$ was obtained independently by measuring the transmission of each optical element with a spectrometer. $I_{s}(k)$ was not obtained directly due to a lack of instrumentation at the present time. However, we estimated $I_{s}(k)$ by measuring the temperature of the source with an optical pyrometer and computing the black body emission at that temperature. (The black body behavior of the source was assured by the manufacturer.) We then normalized $I_{s}(k)$ to its value at the highest wave number. The normalized ab-

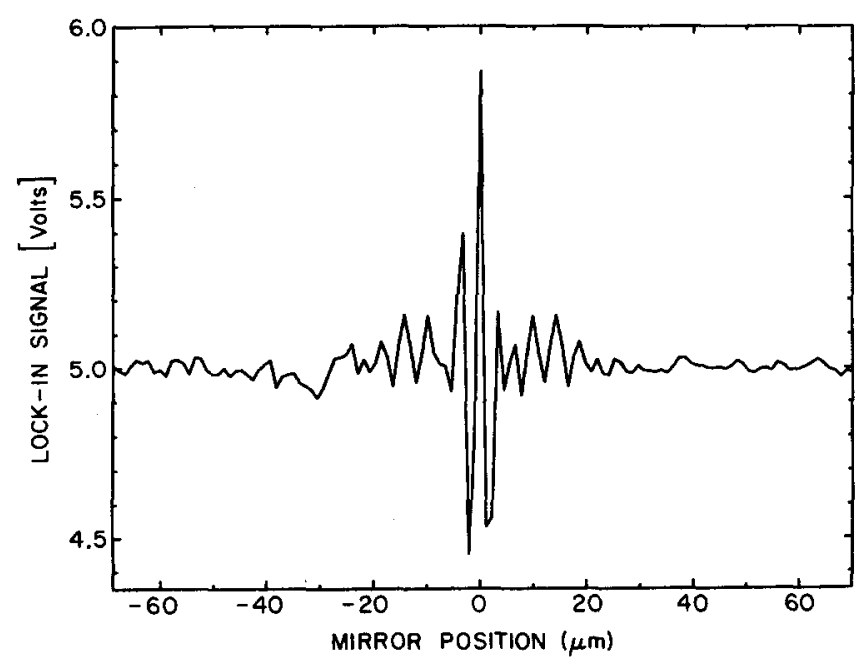

FIG. 4. The amplitude of the scattered light modulation, obtained with a lock-in amplifier, as a function of the interferometer mirror position for a $24.4 \%$ (by mass) $\left(\mathrm{NH}_{4}\right)_{2} \mathrm{SO}_{4}-\mathrm{H}_{2} \mathrm{O}$ particle, $3.14 \mu \mathrm{m}$ radius.

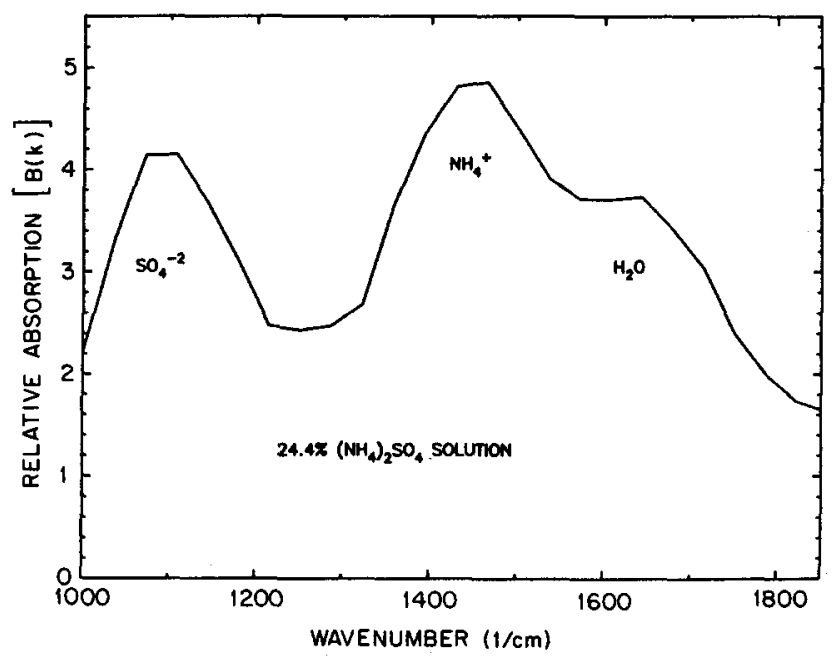

FIG. 5. The convolution of the instrument line function and the particle absorption spectrum $B(k)$ as a function of wavelength, obtained by a discrete FFT inversion of the data shown in Fig. 4.

sorption of the particle, shown in Fig. 6, was obtained by dividing $B(k)$ in Fig. 5 by the instrument line function. Therefore, the spectrum in Fig. 6 exhibits the wave number behavior of the particle absorption. The peaks identified in Fig. 6 are the sulfate, the ammonium, and the water lines. Note that the water peak, more pronounced in Fig. 5, is now a shoulder on the side of the ammonium peak. The theoretical resolution of this scan was $\approx 70 \mathrm{~cm}^{-1}$, however, fluctuations in $\beta$ during the time of the scan are most probably responsible for the enhanced width of the peaks shown by the data. The position of these peaks agree with previous work on a levitated particle, ${ }^{13}$ and also with data taken on bulk solutions. ${ }^{21,22}$ Both the sulfate and the ammonium peaks are positioned above a broad absorption base line. This is consistent with the data for water and ammonium sulfate absorption presented by $\mathrm{Volz}^{22}$ and is considered to be associated with hydrogen bonding.

To show the effect of the water concentration we repeated the experiment at a smaller relative humidity (a higher salt concentration). At this condition the salt weight fraction was $63.3 \%$ and the particle radius was $2.25 \pm 0.02 \mu \mathrm{m}$.

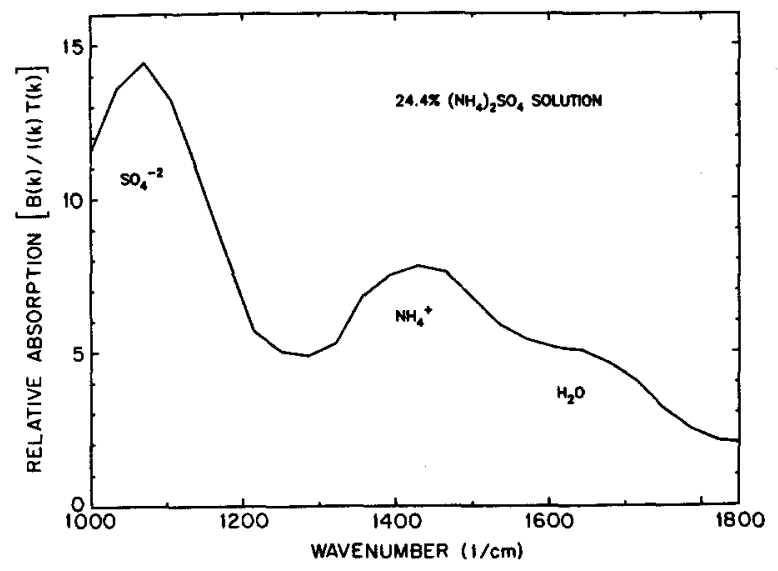

FIG. 6. The relative absorption spectrum $B(k) / I_{s}(k) T(k)$ of the $\left(\mathrm{NH}_{4}\right)_{2} \mathrm{SO}_{4}$ particle in Fig. 5. 
The result is shown as the solid line in Fig. 7, where for comparison we also include the spectrum from Fig. 6 (shown as the dashed line). The effect of the different particle radii, as well as different amplification factor $\beta$ was accounted for in Eq. (11). The effect of the reduced amount of water in the drop on the spectrum is clearly seen by the fall of the absorption base line near $1280 \mathrm{~cm}^{-1}$. The $63.3 \%$ $\left(\mathrm{NH}_{4}\right)_{2} \mathrm{SO}_{4}$ solution is highly concentrated (13 molal), where the ratio of water molecules to salt ions is roughly 1.4 to 1 . In contrast, the corresponding ratio at the solute weight fraction of $24.4 \%$ is 7.6 to 1 . Due to the substantial decrease in the amount of water in the particle, one would expect a reduction in the degree of hydrogen bonding in the concentrated solution. By looking at the water peak in Fig. 7, it is apparent that its position was shifted upward in the supersaturated state. This may be due to the greater average proximity of the water molecules to the ions in the solution which can restrict the water bending mode. Note also that the saturation point for ammonium sulfate at room temperature is about 5 molal, and thus the spectrum shown in Fig. 7 represents that of a supersaturated solution. This is apparently the first reported spectrum for an ammonium sulfate solution at this degree of saturation.

As mentioned above, the resolution of the spectra in Figs. 5 and 6 was $\approx 70 \mathrm{~cm}^{-1}$. Increasing the resolution by moving the interferometer mirror a greater distance did not prove useful due to the poor signal-to-noise ratio in the wings of the interferogram. An important contribution to this noise is the particle size drift due to temperature variations in the chamber during the run. The main causes of these variations are the heating of the chamber by the IR source, and the heating or cooling of the chamber due to changes in the ambient temperature. These temperature changes alter the relative humidity in the chamber which forces the particle to change its size accordingly. The manifestation of this drift is that $\beta$ is not absolutely constant during the experiment. Since the scattered light signal is directly proportional to $\beta$, the temperature fluctuations contribute strongly to the noise in the interferogram.

The drift problem mentioned above could be reduced by using smaller particles. The advantage of using smaller particles is that their resonances are wider. Although wider resonance implies that the amplification factor $\beta$ is smaller (the resonance curve is less steep), the scattered light becomes less sensitive to small drifts in the particle size. To overcome the problem of decrease in modulation signal the IR chop rate can be reduced. Since the particle relaxation time constant decreases with the square of the radius, ${ }^{13,14}$ the response time of the detector (the particle in our case) improves. The faster response assures that the particle returns back to its original size quickly thereby yielding a cleaner size modulation and lower noise.

\section{CONCLUSIONS}

We have reported here a new method based on Fourier transform spectroscopy to obtain a complete molecular spectrum of a single suspended aqueous microparticle. The first interferogram of a $3.14 \mu \mathrm{m}$ radius ammonium sulfate droplet has been presented. We have also presented a spectrum of a particle in a highly supersaturated state. The differences between the two spectra show the effect of reduced hydrogen bonding in aqueous solutions.

\section{ACKNOWLEDGMENTS}

This research was supported by U. S. Environmental Protection Agency Grant No. R-810857 and by gifts from General Motors Corporation and Mobil Oil Corporation. S. Arnold was supported by a Chevron Visiting Professorship at the California Institute of Technology and by National Science Foundation Grant No. ATM-8413574. The authors thank Anthony B. Pluchino of the Aerospace Corporation and Ralph Waniak of Advanced Kinetics for helpful advice during this work.

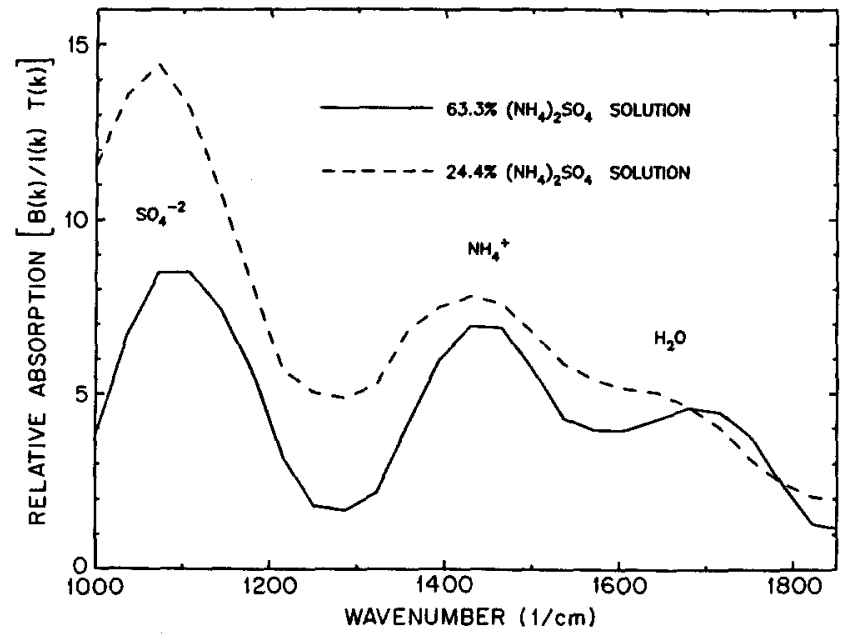

FIG. 7. The effect of a reduction in the particle water content on the absorption spectrum, obtained for 13 molal (solid line) and 2.5 molal (dashed line) $\left(\mathrm{NH}_{4}\right)_{2} \mathrm{SO}_{4}$ particles.
${ }^{1}$ R. H. Frickel, R. E. Shaffer, and J. B. Stamatoff, Technical Report ARCSL-TR-77041 U. S. Army Armament Research and Development Command, Chemical Systems Laboratory, Aberdeen Proving Grounds, Maryland (1955).

${ }^{2}$ R. F. Weurker, H. Shelton, and R. V. Langmuir, J. Appl. Phys. 30, 342 (1959).

${ }^{3}$ E. J. Davis, Langmuir 1, 379 (1985).

${ }^{4}$ E. J. Davis, P. Ravindran, and A. K. Ray, Adv. Colloid Interface Sci. 15, 1 (1981).

${ }^{5}$ G. O. Rubel, J. Colloid Interface Sci. 85, 549 (1982).

${ }^{6} \mathrm{G}$. O. Rubel, paper 5F4, Ann. Conf. AAAR, Albuquerque, NM (1985).

${ }^{7}$ M. C. Cohen, R. C. Flagan, and J. H. Seinfeld (submitted).

${ }^{8}$ L. M. Folan, S. Arnold, and S. D. Druger, Chem. Phys. Lett. 118, 322 (1985).

${ }^{9}$ R. Thurn and W. Kiefer, Appl. Opt. 24, 1515 (1985).

${ }^{10}$ R. E. Preston, T. R. Letteri, and H. Semerjian, Langmuir 1, 365 (1985).

${ }^{11}$ S. Arnold, M. Neuman, and A. B. Pluchino, Opt. Lett. 2, 4 (1984).

${ }^{12}$ S. Arnold and A. B. Pluchino, Appl. Opt. 21, 4199 (1982).

${ }^{13}$ S. Arnold, E. K. Murphy, and G. Sageev, Appl. Opt. 7, 1048 (1985).

${ }^{14}$ M. R. Querry, R. V. Waring, W. E. Holland, L. M. Earls, M. D. Herrman, W. P. Nijm, and G. M. Hale, J. Opt. Soc. Am. 64, 39 (1974).

${ }^{15}$ G. Sageev, R. C. Flagan, J. H. Seinfeld, and S. Arnold, J. Colloid Interface Sci. 113, 421 (1986). 
${ }^{16}$ R. G. Bell, Introductory Fourier Transform Spectroscopy (Academic, New York, 1972),

${ }^{17}$ G. Sageev, J. H. Seinfeld, and R. C. Flagan, Rev. Sci. Instrum. 57, 933 (1986).

${ }^{18}$ S. Arnold and L. M. Folan, Rev. Sci. Instrum. 57, 2250 (1986).

${ }^{19}$ G. Mie, Ann. Phys. 25, 377 (1908)
${ }^{20}$ A. Ashkin and J. M. Dziedzic, Phys. Rev. Lett. 38, 1351 (1977).

${ }^{21}$ E. E. Remsberg, Appl. Opt. 12, 1389 (1973).

${ }^{22}$ F. E. Volz, Appl. Opt. 12, 565 (1973).

${ }^{23}$ M. R. Querry, R. V. Waring, W. E. Holland, G. M. Hale, and W. P. Nijm, J. Opt. Soc. Am. 62, 849 (1972). 